\title{
Fire Disaster Monitoring Network Localization algorithm Based on RSSI Ranging technology
}

\author{
CHANG Bo ${ }^{1, a}$, ZHANG Xin-rong ${ }^{2,3, b}$, XV Bao-guo ${ }^{3, c}$ \\ ${ }^{1}$ Faculty of Electronic Information Engineering, HuaiYin Institute of Techenology, huaian 223003, \\ China \\ ${ }^{2}$ Faculty of Automation Engineering, HuaiYin Institute of Technology, huaian 223003, China \\ ${ }^{3}$ School of Internet of Things Engineering, JiangNan University, wuxi 214122, China \\ ammm33534@sohu.com, bnn33@163.com, xbg@jiangnan.edu.cn
}

Keywords: fire disaster monitoring, wireless sensor network (WSN), RSSI ranging, RLS algorithms, Localization

\begin{abstract}
In order to understand the environment and the situation of fire disaster and improve the efficiency of putting out the fire and emergency rescue, aiming at the actual demand of monitoring node location of fire disaster, a new recursive LS (RLS) localization method for monitoring fire environment based on wireless sensor networks is proposed, which method is based on dynamic path loss index ranging algorithm. The path loss exponent is obtained periodically, then comparing the relative error coefficients of each reference node; finally the coordinates of the monitoring nodes are estimated using the RLS localization algorithm based on the forgetting regulator. The system can realize the fire automatic monitoring and location, real-time collecting and processing these data such as the temperature, humidity and smoke concentration etc. The simulation results show that the method can meet the requirements of the location of the fire environment monitoring system. Compared with the conventional LS algorithm, the proposed localization algorithm has higher accuracy and lower computational complexity.
\end{abstract}

\section{Introduction}

WSN (Wireless sensor network) capable of sensing, collecting, transmitting and processing environmental data in the monitoring area [1] and is able to implement low power unattended monitoring in poor areas that people can't reach [2]. Wireless network fire monitoring system can reduce the damage to buildings. The self-organization of wireless network makes the disaster monitoring system can be made in a short time, and can achieve the monitoring of dangerous situations without human intervention [3]. Dynamic monitoring of the fire scene using mobile monitoring nodes can not only reduce the number of monitoring points, but also increase the information acquisition range of the network, and search for effective information.

General location algorithm is based on distance measurement. The common ranging techniques include time of arrival (TOA), time difference of arrival (TDOA), angle of arrival (AOA) and received signal strength indicator (RSSI). RSSI ranging is concerned because there is no need to add additional hardware devices, low power consumption and low cost, but it is greatly influenced by environment, so nonlinear modeling of wireless signal propagation leads to the problem of nonlinear estimation [4]. The RF wireless communication modeling ranging with additional redundant information is advantageous to the improvement of the location accuracy, so the RSSI is a kind of cost-effective ranging and locating solution in appropriate application conditions. Three methods of path loss index calculation hav e been proposed based on the establishment of the signal propagation model [5]. It is pointed out that the path loss exponent can be calculated without distance measurement, and it is proposed to meet the needs of positioning and online real-time correction in the references [6]. Two methods of model parameters calculation are proposed in the references [7] on the basis of RF signal propagation modeling, that is, least square algorithm and average handover algorithm. On the basis of the traditional three edge locating algorithm, the paper [8] proposes an 
improved localization algorithm using the error correction method. In the paper [9], a new method based on multi-level energy RSSI is proposed, which takes into account the intrinsic connection between the intensity of the signal emission and the RSS value.

In this paper, a dynamic acquisition ranging algorithm based on path loss factor is proposed, which based on the actual demand of fire environment localization. The accuracy of the algorithm can meet the requirements of the system, and can be applied to the WSN monitoring node location in the fire monitoring system.

\section{Monitoring Node Ranging Based on RSSI}

Wireless Signal Propagation Model. In the environment of forest fire monitoring system, due to multipath, diffraction and obstacles occlusion factors, the modeling of the RF signal becomes complex. In the environment of limited power consumption, the use of the logarithmic distance path loss model is appropriate. RSSI values can be expressed as:

$$
P_{R}(d)=P+G-P_{L}(d)
$$

where $P$ is a node transmit power, $G$ is a node antenna gain, $P_{L}(d)$ is a RF power loss after a distance of $d$. Signal propagation modeling can also be written as the following expression:

$$
P_{R}(d)=P_{R}\left(d_{0}\right)-10 n \lg \left(d / d_{0}\right)+X_{\sigma}
$$

where $n$ is the path loss factor; $d_{0}$ is the reference distance of signal propagation, which value is $1 \mathrm{~m}$ normally, $P_{R}\left(d_{0}\right)$ is the received signal strength of $d_{0}, X_{\sigma}$ is the Gaussian random variables which mean is 0 and standard deviation range is $4 \sim 10$.

Dynamic Acquisition of Path Loss Exponent. The location of the reference node is known, so that the signal intensity between the reference nodes can be obtained by periodic dynamic measurement, and the path loss exponent can be obtained by using the logarithm distance path loss model. If the reference node $R_{0}$ receives the value of other two reference nodes, $R_{1}$ and $R_{2}$. There are the following formulas:

$$
P_{R}\left(d_{1}\right)=P_{R}\left(d_{0}\right)-10 n \lg \left(d_{1} / d_{0}\right)+X_{\sigma}, \quad P_{R}\left(d_{2}\right)=P_{R}\left(d_{0}\right)-10 n \lg \left(d_{2} / d_{0}\right)+X_{\sigma}
$$

From the Eq. 3, the value of $n$ is obtained:

$$
n=\frac{P_{R}\left(d_{2}\right)-P_{R}\left(d_{1}\right)}{10 \lg \left(\frac{d_{1}}{d_{0}}\right)-10 \lg \left(\frac{d_{2}}{d_{0}}\right)}
$$

where $P_{R}\left(d_{1}\right)$ is the value between $R_{0}$ and $R_{1}, P_{R}\left(d_{2}\right)$ is the value between $R_{0}$ and $R_{2}, d_{1}$ is the actual distance between $R_{1}$ and $R_{0}, d_{2}$ is the actual distance between $R_{2}$ and $R_{0}, n$ is the path loss exponent. The more obstacles is, the more $n$ is. From the Eq. 4, the value of dynamic path loss index $n$ corresponding to the actual environment is obtained from the obtained $P_{R}(d)$.

Method to Reduce the Error of Ranging. Using RF signal propagation attenuation model, the measurement error of $P_{R}(d)$ value of the reference node can be obtained by comparing the measured distance and the actual distance. Therefore, if the measurement error of the reference node is considered, the interference of the noise in the network can be reduced.

Considering the two dimensional case, the reference node is assumed for $R_{i}, i=1,2, \ldots, n$, where $n$ is the number of reference nodes participating in the correction. $R_{0}\left(x_{0}, y_{0}\right)$ is the reference node to be corrected. The actual distance from $R_{0}\left(x_{0}, y_{0}\right)$ to $R_{i}\left(x_{i}, y_{i}\right)$ is ri. The distance measured by $P_{R}(d)$ is $d_{i}$, $i=1,2, \ldots, n$. The relative error of ranging is:

$$
\mu_{i}=\left(r_{i}-d_{i}\right) / d_{i}
$$


The relative error correction factor for the weighted ranging of the reference node $R_{i}$ is:

$$
\mu_{W}=\sum_{i=1}^{n} \frac{r_{i} d_{i}}{d_{i}^{2} \sum_{i=1}^{n} 1 / d_{i}}
$$

The $\mu_{\mathrm{w}}$ reflects the measurement error of the reference node $P_{R}(d)$. Taking into account the weight of different $P_{R}(d)$, with the increase of distance, the greater the distance error caused by the deviation of $P_{R}(d)$ is, the smaller the decision power of the correction factor is. The correction distance of reference node is:

$$
d_{u i}^{c}=d_{u i}\left(1+\mu_{i}\right)
$$

where $d_{u i}$ is the distance between the node and the reference node $R_{i}, d_{u i}^{c}$ is the correction distance between the monitoring node and the reference node $R_{i}$, and the $\mu_{\mathrm{w}}$ is the relative error correction factor of the weighted ranging of the reference node $R_{i}$.

\section{Monitoring Node Localization Algorithm}

Supposing there are $N$ reference nodes in the network marked as $R_{i}(x i, y i)$. The distance from these nodes to the unknown node $U\left(x_{i}, y_{i}\right)$ is $d_{i} . i=1,2, \ldots, N$,. The measurement equations are expressed as:

$\hat{d}_{l}=d_{i}+v_{i}, i=1,2, \ldots, N$

where $d_{i}=\sqrt{\left(x_{i}-x\right)^{2}+\left(y_{i}-y\right)^{2}}, \quad v_{i}$ is measurement error. The Eq. 8 can be abbreviated as:

$h=G \theta+v$

where,

$G=\left[\begin{array}{ccc}-2 x_{1} & -2 y_{1} & 1 \\ \vdots & \vdots & \vdots \\ -2 x_{N} & -2 y_{N} & 1\end{array}\right]$

$h=\left[\hat{d}_{1}^{2}-\left(x_{1}^{2}+y_{1}^{2}\right), \ldots, \quad \hat{d}_{N}^{2}-\left(x_{N}^{2}+y_{N}^{2}\right)\right]^{T}$

$v=\left[\begin{array}{lll}2 \hat{d}_{1} v_{1}-v_{1}{ }^{2}, \ldots, & 2 \hat{d}_{N} v_{N}-v_{N}{ }^{2}\end{array}\right]^{T}$

$\theta=\left[\begin{array}{lll}x & y & R\end{array}\right]^{T}$

$R=x^{2}+y^{2}$

Then the solution of $L S$ is:

$$
\hat{d}_{1}=\left(G^{T} G\right)^{-1} G^{T} h
$$

The solution of WLS is:

$$
\hat{d}_{1}=\left(G^{T} W G\right)^{-1} G^{T} W h
$$

where, $W=\operatorname{cov}^{-1}(v)$ is the weighted matrix. In the practical application, the $W$ can be set to the unit array if the error statistics are unknown.

The least square estimation method in Eq. 10 is unable to meet the real-time requirements of the current application, but the recursive algorithm only needs to retain the previous state values and obtain the current observation data, which not only save the storage space, and reduce the complexity of computation, but also improve the real-time performance of the algorithm. Let $\hat{\theta}_{k}$ be used for the estimation of the least square algorithm using the previous k observation data. According to the Eq. 9, the measurement process is modeled as: 


$$
h_{k}=G_{k} \theta_{k}+v_{k}
$$

The error cost function can be written as:

$$
J\left(\theta_{k}\right)=\frac{1}{2} \sum_{k=1}^{n}\left(h_{k}-G_{k}^{T} \theta_{k}\right)^{2}
$$

The value of $\theta_{k}$ to minimize the cost function is the estimated value of $\hat{\theta}_{k}$.

$$
\hat{\theta}_{k}=\left(\sum_{k=1}^{n} G_{k}^{T} G_{k}\right)^{-1}\left(\sum_{k=1}^{n} G_{k} h_{k}\right)
$$

As the previous measurement error information has little effect on the current parameters, in order to reduce the computational burden of error information, an index weighting factor based on the RLS estimation algorithm is introduced, that is, forgetting factor, so as to form the RLS estimation algorithm with forgetting factor. Eq. 13 can be rewritten as:

$$
J\left(\theta_{k}\right)=\frac{1}{2} \sum_{k=1}^{n} \lambda^{n-k}\left(h_{k}-G_{k}^{T} \hat{\theta}_{k}\right)^{2}, \lambda \in(0,1)
$$

where, $\lambda$ is the forgetting factor. The RLS algorithm with forgetting factor is derived by reorganizing:

$$
\hat{\theta}_{k}=\hat{\theta}_{k-1}+C_{k}\left[h_{k}-G_{k}^{T} \hat{\theta}_{k-1}\right]
$$

where $C_{k}$ is the recursive gain matrix, we have:

$$
C_{k}=Q_{k-1} G_{k}\left[\lambda I+G_{k}^{T} Q_{k-1} G_{k}\right]^{-1}
$$

where $Q_{k}$ is the covariance matrix, we have:

$$
Q_{k}=\left[I-C_{k} G_{k}^{T}\right] Q_{k-1} / \lambda
$$

\section{Simulation Model and Test Data}

In the Gauss semi Markov entity motion model, it is thought that movement is smooth and can overcome the shortcomings of the emergency stop, so the movement is more realistic. The movement speed $v_{k}$ and the direction $\phi_{k}$ of the monitoring nodes at the time of the $\mathrm{k}$ are respectively as follows:

$$
v_{k}=\xi v_{k-1}+(1-\xi) \bar{v}+\sqrt{1-\xi^{2}} \tilde{v}_{k-1}, \quad \phi_{k}=\xi \phi_{k-1}+(1-\xi) \bar{\phi}+\sqrt{1-\xi^{2}} \tilde{\phi}_{k-1}
$$

where, $\xi$ is the parameters related for the motion, and its range is $[0,1]$. The average velocity and the average movement direction are indicated by $\bar{v}$ and $\bar{\phi}$ respectively. The $\tilde{v}_{k-1}$ is a Gauss random variable, which mean is 0 , the standard deviation is $\sigma_{v}$ and has nothing to do with $v_{k}$. The $\bar{\phi}_{k-1}$ is a Gauss random variable, which mean is 0 , the standard deviation is $\sigma_{\phi}$ and has nothing to do with $\phi_{k}$. At the moment of $K$, the expression of node coordinate is as follows:

$$
x_{k}=x_{k-1}+v_{k-1} \times \cos \left(\phi_{k-1}\right), \quad y_{k}=y_{k-1}+v_{k-1} \times \sin \left(\phi_{k-1}\right)
$$

In the fixed experimental area (about $100 \mathrm{~m}^{*} 100 \mathrm{~m}$ ), the mobile monitoring nodes move in the experimental area according to the Gauss semi Markov model and the model parameters are set: $\xi=0.6, \bar{v}=1 \mathrm{~m} / \mathrm{s}, \bar{\phi}=\pi / 2$. The starting position of the monitoring node is set to $(40 \mathrm{~m}, 40 \mathrm{~m})$, and a positioning is finished every $5 \mathrm{~s}$, so there are 15 times in total for the algorithm performance testing. The moving track of the mobile monitoring node is shown in Fig. 1. 


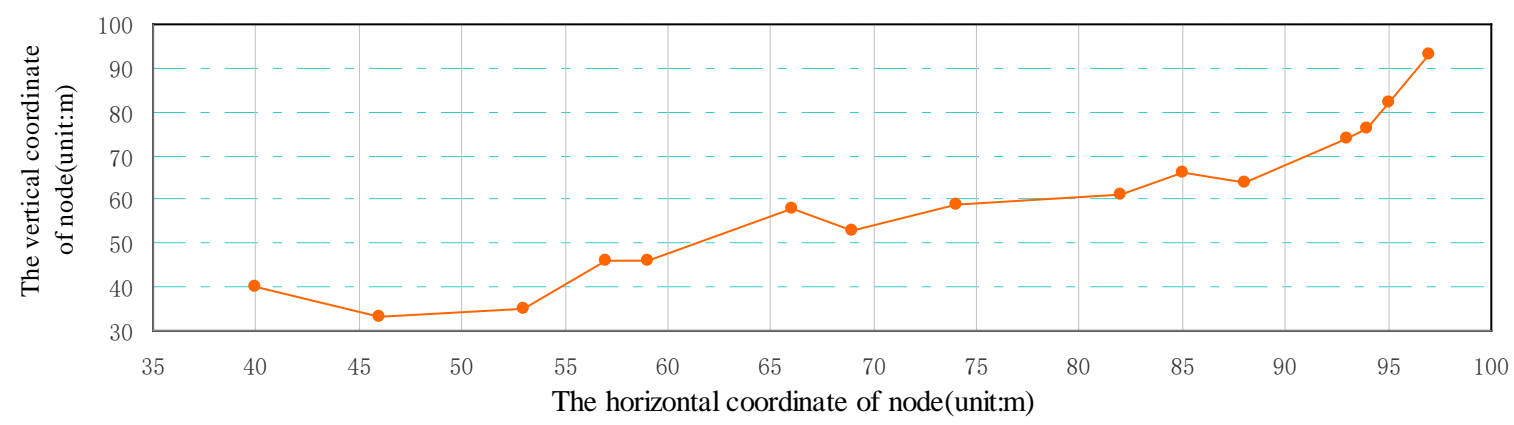

Fig. 1 The moving track of the mobile monitoring node

\section{Simulation Experiment and Analysis}

The location error of the nodes in the network is defined as:

$$
E_{k}=\frac{\left\|p_{i}-z_{i}\right\|}{R}
$$

where $R$ is the communication radius. $p_{i}=\left[\begin{array}{ll}p_{x i} & p_{y i}\end{array}\right]^{T}$ is the final estimate position of node $\mathrm{i}$, and $z_{i}=\left[\begin{array}{ll}z_{x i} & z_{y i}\end{array}\right]^{T}$ is the true position of node $i$. The average location error $E_{a}$ of the nodes in the network is defined as:

$$
E_{a}=\frac{\sqrt{\sum_{i=1}^{N}\left\|p_{i}-z_{i}\right\|^{2}}}{N R}
$$

where $i=1,2, \ldots, N, N$ is the number of unknown nodes in the network.

Using the proposed algorithm in this paper, we analyzed the test data of the 15 groups. The measurement noise is considerd as $X_{\sigma}(0,5)$. The 100 nodes are randomly distributed in the region of $100 \mathrm{~m} \times 100 \mathrm{~m}$, the node's communication radius is $40 \mathrm{~m}$ and the reference node number is 20 . In order to reduce the random error, the results are the mean of the 100 times simulation results under the same parameters. The positioning error of the test data is shown in Fig. 2.

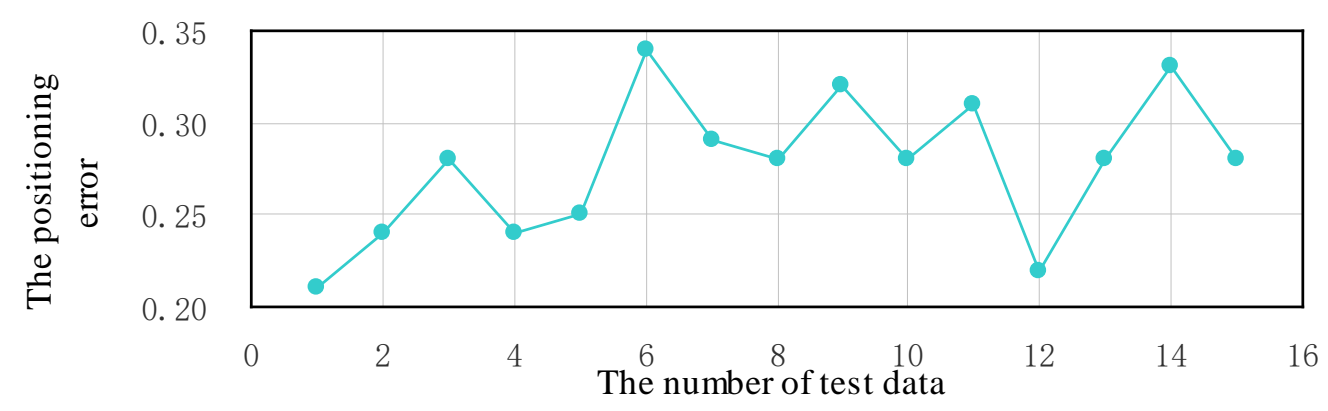

Fig. 2 The positioning error of the test data

From the Fig. 2, We can see that the maximum error is 0.34 and the minimum value is 0.21 . Because the 10 reference nodes are spaced along the upper and lower boundaries, so the location error of the edge of the experimental area is large and the overall positioning effect is good. If we deploy some reference nodes to the edge of the test area, we can further improve the positioning effect.

Selecting the different noise variance to position the test data, the reference node value is 20 and the number of nodes is 100. The positioning results are shown in Fig. 3. 


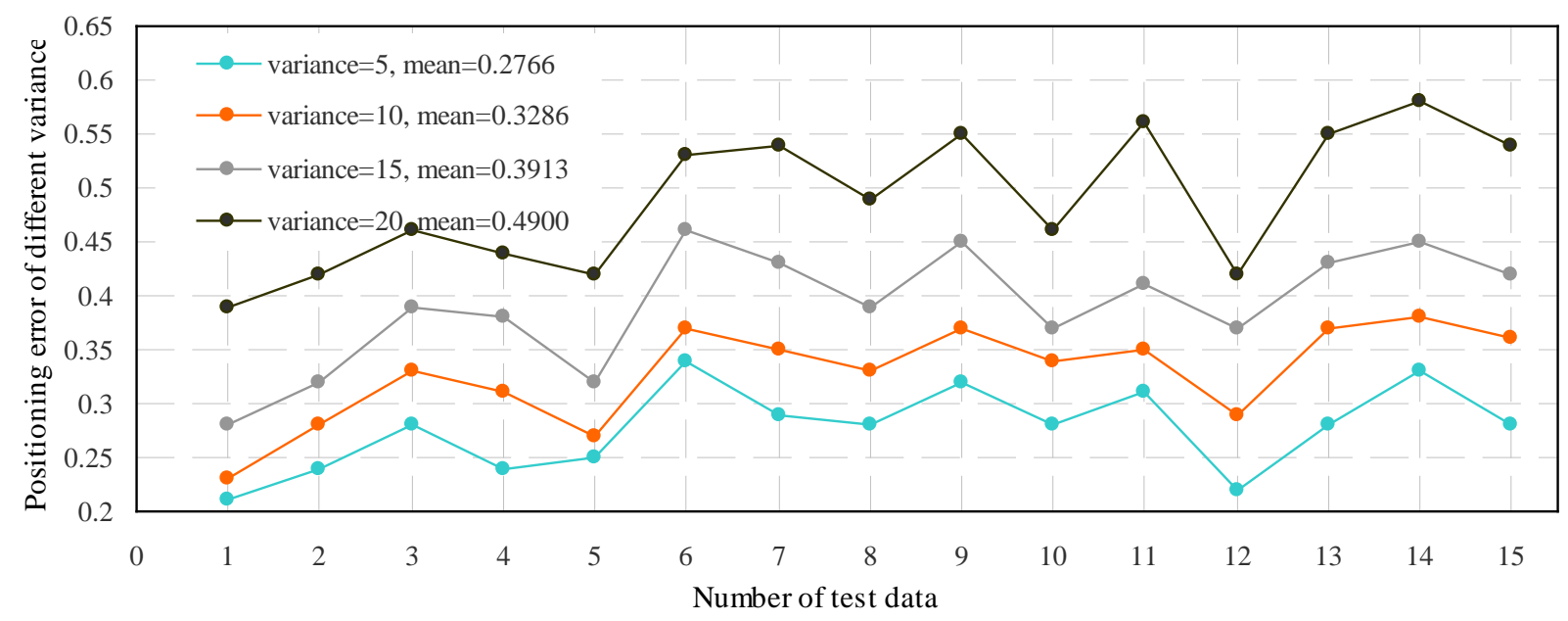

Fig.3 Effect of measurement noise on positioning error

From the Fig.3, we can see that the positioning error of the test data is increasing with the increase of the variance of the Gauss random variables, but the positioning error of the edge of the test area is not obvious. The mean value of the positioning error is $0.2766,0.3286,0.3913$ and 0.4900 respectively in four cases, which shows that the proposed algorithm has good environment adaptability. Compared with the conventional LS algorithm, the mean value of the two algorithms is 0.2413 and 0.2851 respectively, and the average running time of the algorithm is $0.2475 \mathrm{~s}$ and $0.2946 \mathrm{~s}$ respectively at the same ranging error and the same reference node number (25).

\section{Summary}

In this paper, a recursive least square algorithm based on RSSI distance measurement and relative error correction is presented. The algorithm is used to monitor the fire disaster, and to obtain the accurate position information of the nodes is obtained The accuracy of this algorithm can meet the requirements of fire monitoring system, and the computation complexity and communication overhead are small, especially it can effectively resist noise interference, which the WSN monitoring system can be applied to the monitoring node location in fire monitoring system. The simulation results show that the proposed algorithm in this paper has good performance in both the accuracy and the computational complexity compared with the LS localization algorithm.

\section{References}

[1] Zeng X Z, Liu G, Zheng D P, et al. Study and development of a field information acquisition system based on wireless technique[C] // Actual Tasks on Agricultural Engineering, Opatija, CROATIA, 2006: 371 377.

[2] Sun Zhongfu, Du K M, Han H F, et al. Design of a telemonitoring system for data acquisition of livestock environment[C] // Livestock Environment VIII -Proceedings of the 8th International Symposium, Iguassu Falls, Brazil: ASABE, 2008: 995 1000.

[3] Young Neil.Fire Dctection and Alarm Systems[J].Fire Prevention and Fire Engineers Journals.2004, 4:53-55.

[4] Li Z, Wang N, Hong T. Radio Path - Loss Modeling For A 2.4 Ghz In - Field Wireless Sensor Network[J]. Transactions of the ASABE, 2010, 53(2): 1-10.

[5] S. Srinivasa, M. Haenggi. Path loss exponent estimation in large wireless net-works [C]. Information Theory and Applications Workshop, San Diego, CA,2009: 124-129.

[6] G. Mao, B. D. 0. Anderson. Path loss exponent estimation for wireless sensor network 
localization[J]. Computer Networks 2007, 51(10): 2467-2483.

[7] N. Benvenuto, F. Santucci. Comparison between least squares path loss estimation and averaging for handover algorithms[C]. Vehicular Technology Conference on Mobile Technology for the Human Race, Atlanta, GA, 1996:1326-1330.

[8] Gao Guosheng, Chen Junjie. Self calibration and localization algorithm of beacon node based on RSSI ranging [J]. Measurement and Control Technology, 2009, 28(8): 93-97.

[9] J. Y. Fang, H. C. Chu. A multiple power-level approach for wireless sensor network positioning[J]. Computer Networks 2008, 52(16): 3101-3118. 